\title{
Regeneración vegetal en la caldera del volcán Reventador en la cordillera subandina del norte de la Amazonía Ecuatoriana
}

\section{Vegetation regeneration on the Reventador volcano calderain, the subandean mountain chain, north Ecuadorian Amazon}

\author{
Pablo Lozano ${ }^{1,2^{*}}$, Rainer W. Bussmann ${ }^{3}$, Gwendolyn Peyre ${ }^{4}$ \& Félix Grefa ${ }^{1}$ \\ ${ }^{1}$ Herbario ECUAMZ, Universidad Estatal Amazónica, Pastaza 160101, Ecuador. \\ ${ }^{2}$ Departamento de Ciencias de la Vida, Universidad Estatal Amazónica, Pastaza 160101, Ecuador. \\ ${ }^{3}$ Department of Ethnobotany, Institute of Botany, Ilia State University, Tbilisi, Georgia, rainer.bussmann@iliauni.edu.ge \\ ${ }^{4}$ Dpto. de Ingeniería Civil y Ambiental, Universidad de los Andes, Cra. 1E \#19a-40, 111711, Bogotá, Colombia. gf.peyre@uniandes.edu.co \\ *Correspondencia: plozano@uea.edu.ec
}

\section{Resumen}

L as frecuentes erupciones con coladas de lavas en la caldera del volcán Reventador han favorecido a un terreno fértil para estudios de sucesión en diferentes paisajes y edades. Se establecieron un total 25 parcelas $(5 \times 5 \mathrm{~m})$ en caso de vegetación herbácea o arbustiva baja y de $100 \mathrm{~m}^{2}(10 \mathrm{x} 10 \mathrm{~m})$ para vegetación arbustiva alta; se empleo el método fitosociologico para recensar la vegetación vascular (identificación y cobertura) y no vascular. Se identificaron 12 especies endémicas en nueve familias, donde se destacan las familias Bromeliaceae y Melastomataceae, cuatro especies endémicas son catalogados En Peligro, tres Vulnerables, dos en Preocupación Menor, una Casi Amenazada, una No Evaluada, y una Datos Insuficientes. Se uso el programa TWINSPAN para la caracterización de los diferentes tipos de unidades de paisaje y asociaciones, definiéndose dos Unidades Vegetales, tres subunidades y siete asociaciones, la Unidad I (2094-2391 msnm), compuesta de tres estratos: herbáceo, arbustivo bajo y arbustivo alto, mientras que la Unidad II (2152-2213 msnm), mayoritariamente compuesta por especies pioneras de estratos bajos con dominancia de briofitas y líquenes. Se registraron 112 especies de plantas vasculares, pertenecientes a 70 géneros en 42 familias botánicas, con un rango de cobertura vegetal de 80 a $100 \%$. Las especies con mayor diversidad relativa fueron: Epidendrum con 8 especies (7.27\%), Elleanthus con 5 especies (4.46\%), Blechnum con 4 especies (3.57), Elaphoglossum, Lycopodium, Axinea y Psychotria con 3 especies $(2.69 \%)$ respectivamente. La notable presencia de endemismo 12 especies en un espacio reducido, corresponde a que la caldera antigua del volcán Reventador, se encuentra rodeada por un bosque montano primario; que abastece de recursos para su repoblamiento, encontrando nichos que favorecen a la especiación. El presente estudio se constituye la primera descripción botánica de la sucesión vegetal en la caldera del Reventador, aportando los primeros datos y registros botánicos para el país.

Palabras clave: Volcán Reventador, Sucesión vegetal, Endemismo, Agrupaciones floristicas
Rec.: 16.05.2019. Acept.: 03.01.2020 Publicado el 30 de junio de 2020

Abstract

$\mathrm{I}^{\mathrm{n}}$ the caldera of Reventador volcano frequent eruptions with lava flows have provided fertile ground for succession studies in different landscapes and ages. A total of 25 plots, were established. Plot sizes were $5 \times 5 \mathrm{~m}$ to study the low herbaceous or shrubby vegetation, and $10 \times 10 \mathrm{~m}$ for tall shrubby vegetation. A phytosociological method was used to assess vascular (identification and coverage) and non-vascular vegetation. Twelve endemic species belonging to nine families were identified, with Bromeliaceae and Melastomataceae standing out. Four endemic species are currently listed as Endangered, three as Vulnerable, two of Minor Concern, one as Near Threatened, one as Not Assessed, and one had Insufficient Data. The TWINSPAN program package was used to characterize the different types of landscape units and associations, defining two vegetation units, three sub-units and seven associations, Unit I (2094-2391 masl), was composed of three strata: herbaceous, shrubby low and high shrub, while Unit II (2152-2213 masl), was mostly composed of pioneer species of low strata with dominance of bryophytes and lichens. One hundred twelve species of vascular plants were registered, belonging to 70 genera in 42 botanical families, with a range of plant cover of 80 to $100 \%$. The species with the greatest relative diversity were: Epidendrum (8 species, 7.27\%), Elleanthus (5 species, 4.46\%), Blechnum (4 species, 3.57), and Elaphoglossum, Lycopodium, Axinea and Psychotria with 3 species (2.69\%) respectively. The remarkable presence of 12 endemic species in the small space of the old caldera of the Reventador volcano, can be explained by it being surrounded by primary montane forest; which provides resources for its repopulation, given niches that favor speciation. The present study constitutes the first botanical description of the plant succession in the caldera of the Reventador, providing the first botanical data and new records for the country.

Keywords: Reventador Volcano, Plant succession, Endemism, Floristic clusters 


\section{Introducción}

$\mathrm{L}$ as erupciones volcánicas son de las amenazas ambientales naturales más severas del mundo (Walker y del Moral, 2003). De los 3000 volcanes activos en la actualidad, entre 60 y 70 se encuentran en erupción al menos una vez al año, afectando directamente 500 millones de personas. En Ecuador, se encuentran tres volcanes activos particularmente peligrosos, el Tungurahua, el Reventador y el Sangay. El volcán Reventador ubicado en la ladera amazónica de la cordillera subandina norte ecuatoriano tiene una frecuencia de erupción de 16 erupciones entre 1541 al 2002 (Hall et al., 2004) entre otras de los años 20042005, 2007-2008, 2009-2010, 2011-2012, 2013-20142015-2016-2017-2018.

La actividad volcánica afecta primordialmente a la vegetación inmediata e inmóvil, que tiene que reiniciar una sucesión ecológica primaria luego de cada evento eruptivo. Entender las dinámicas de successiones vegetales primarias con el volcanismo es primordial para el conocimiento ecológico de sucesión (Vargas, 1985), y estos estudios son particularmente escasos en la zona tropical. Entre ellos, se encuentran los estudios de dinámica de vegetación sobre lavas en el volcán Krakatoa de la isla de Java (Penzing, 1902), del volcán Rumoka en el Congo, de las islas hawaiianas (Skotberg,1941), del volcán La Soufriere, de la isla San Vicente en las Antillas Menores (Beard, 1945), y de los volcanes Paracutín y Jorullo en México (Eggler, 1959). La edad de los depósitos y la evolución pedogenética tienen una gran influencia en la sucesión, por lo tanto, en la fisonomía, y en la distribución espacial de las diferentes asociaciones sucesionales. No obstante poco se conoce de la recuperación vegetal luego de flujos piroclasticos y lava en el Ecuador, se puede aseverar que sin duda el presenrte estudio corresponde a un estudio pionero del análisis de la vegetación y suceción. Para lograr este objetivo, se compararan diferentes unidades vegetales sobre coladas de lava representando diferentes eventos eruptivos que se organizaran cronológicamente, generando así información útil para la ciencia en áreas naturales poco estudiadas.

\section{Area de estudio}

El volcán Reventador se encuentra en la región nororiental ecuatoriana, en la cordillera subandina, entre las Provincias de Napo-Sucumbíos, cantón El Chaco y Cantón Gonzalo Pizarro, en la parroquia Gonzalo Díaz de Pineda y Reventador respectivamente, al interior del Parque Nacional Cayambe Coca, a $50 \mathrm{~km}$ de la ciudad del Chaco y $30 \mathrm{~km}$ del poblado El Reventador $\left(0^{\circ} 4^{\text {, }}\right.$ 19.74" S; $\left.77^{\circ} 37^{\prime} 6.10^{\prime \prime} \mathrm{W}\right)$. El clima de la zona se categoriza como "Af-Tropical húmedo con ausencia de época seca" en la clasificación climática de Köppen, y se caracteriza por temperaturas comprendidas entre 17$22^{\circ} \mathrm{C}$ y una pluviosidad entre los 3000 hasta $5500 \mathrm{~mm}$ anuales. La vegetación dominante natural del volcán corresponde a un bosque siempreverde montano del Norte de la Cordillera Oriental de los Andes entre los 2000 - 2900 msnm (MAE, 2013).

Po otro lado la historia geológica detallada de este volcán no es muy conocida, debido a su lejanía, la inaccesibilidad y el tiempo nublado persistente. Los primeros registros (seguramente incompletos) de erupción del Reventador datan del fin del siglo XIX, con repetidos eventos en 1898, 1912, 1926-29, 1944, 1958$60,1972-76$, y una accelerada actividad reciente en 2002, 2004-2005, 2007-2008, 2009-2010, 2011-2012, 2013-2014, 2015, 2016, 2017 y 2018. Estas erupciones fueron caracterizadas por flujos piroclásticos, flujos de lava en bloques, de tamaño moderado flujos de escombros (lahares), y caídas de ceniza que depositaron sólo unos pocos milímetros de ceniza en el valle interandino.

\section{Materiales y métodos}

Dara identificar las unidades vegetales a estudiar, se establecieron rangos altitudinales en diferentes puntos de la caldera, tanto al interior como a su alrededor, seleccionando en total 25 puntos de muestreo (Figura 1). En cada punto de muestreo, se establecieron parcelas de $25 \mathrm{~m} 2(5 \times 5 \mathrm{~m})$ en caso de vegetación herbacea o arbustiva baja (dosel inferior a $1.5 \mathrm{~m}$ ) y de $100 \mathrm{~m} 2(10 \times 10 \mathrm{~m})$ en caso de vegetación arbustiva alta (dosel superior a $3.5 \mathrm{~m}$ ). En cada parcela, se empleo el método fitosociologico para recensar la vegetación vascular (identificación y cobertura) y no vascular (cobertura), ver Cuadro 1.

Entre 3 y 4 muestras botánicas de infidividuos fertiles fueron colectadas en las parcelas, guardadas en bolsas ziplocs y luego prensadas y llevadas a herbario para su ulterior identificación y determinación taxonómica de la especie. Ademas, se realizaron fotografías de individuos vivos (macro y micro) para ilustrar los resultados del estudio. Finalmente, para cada parcela, se registraron los siguientes datos ambientales: posición geográfica, altitud, inclinación y temperatura.

Las muestras fueron llevadas al Herbario ECUAMZ de la Universidad Estatal Amzonica, donde fueron procesadas (Bajo la numeración de Lozano No 001 - 0112). Para la identificación taxonómica de cada espécimen se trasladaron todas las muestras al herbario del QCNE (Museo de Ciencias Naturales del Ecuador), y QCA de la Pontificia Universidad Católica, donde los especímenes se compararon con los ejemplares existentes en los herbarios. Los especímenes reposan en 
Cuadro 1. Valores de abundancia - cobertura según escala de Braun-Blanquet

\begin{tabular}{lc}
\hline \multicolumn{1}{c}{ DEFINICIÓN } & VALOR \\
\hline Individuos solitarios con baja cobertura $<1 \%$ & $\mathrm{r}$ \\
Pocos individuos con baja cobertura $<1 \%$ & + \\
$<5 \%$ de cobertura o individuos abundantes con baja cobertura & 1 \\
$5-25 \%$ de cobertura & 2 \\
$25-50 \%$ de cobertura & 3 \\
$50-75 \%$ de cobertura & 4 \\
$75-100 \%$ de cobertura & 5 \\
\hline
\end{tabular}

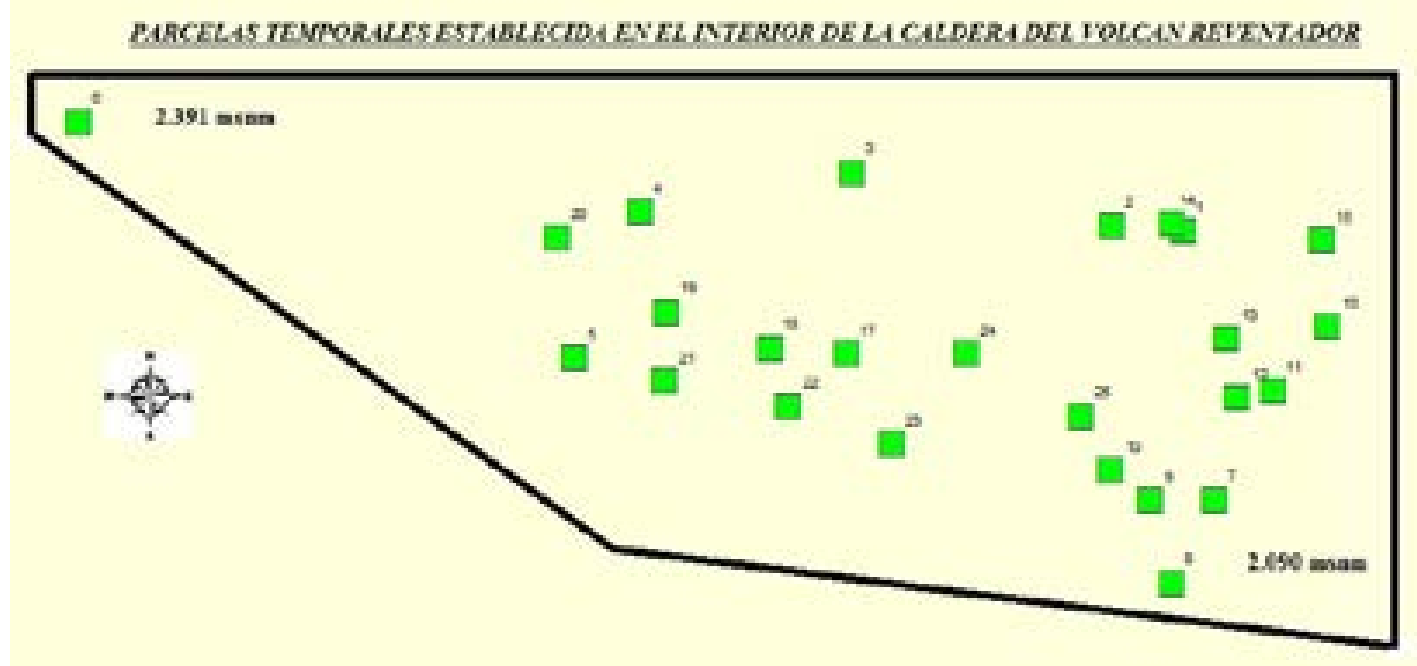

Figura 1. Parcelas temporales establecidas en el interior de la caldera

el herbario ECUAMZ y sus vouchers en los herbarios QCNE y QCA.

Para este trabajo se consideró específicamente el grado de abundancia y frecuencia de las especies en el campo.

\section{Análisis estadísticos}

Para obtener una caracterización de los diferentes tipos de vegetación de la zona que se podrán relacionar con las erupciones, se empleo el programa de agrupación binomial TWINSPAN (Two Way Indicator Species Analysis) Hill \& Smilauer, 2005. TWINSPAN agrupa las parcelas y variables "especies", con base en la mayor similitud en la composición florística, e indica donde se separan los diferentes grupos de parcelas y el valor de probabilidad que los separa (eigenvalor, escala
0-1). Con el análisis TWINSPAN, se procedió a realizar el análisis florístico de los parámetros de diversidad relativa con el uso de la siguiente fórmula:

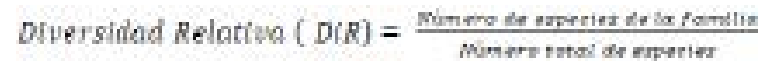

De las muestras colectadas en cada parcela y luego de la identificación, se separaron todos los especimenes endémicos. Se identificó el estado actual de conservación de las plantas raras o en peligro de extinción acorde a Valencia et al., (2000). El grado de endemismo por unidades de paisaje, se determinó; mediante el número de especies por unidad, como se describe (Cuadro 2) a continuación: 
Cuadro 2. Deterinación de especies endémicas

\begin{tabular}{cc}
\hline $\begin{array}{c}\text { Número de Especies } \\
\text { Endémicas }\end{array}$ & Grado de Endemismo \\
$1-2$ & Bajo \\
$3-5$ & Medio \\
$6-10$ & Alto \\
$11-20$ & Muy alto \\
$>21$ & Extremadamente alto \\
\hline
\end{tabular}

\section{Resultados y discusión}

$\mathrm{L}$ a clasificación realizadas en las 25 parcelas, separa y ordena los datos en dos grandes unidades (I y II), la unidad I se separa en tres sub-unidades y siete asociaciones vegetales, la unidad II consta de una asociación. El dendrograma señala los niveles de separación que fueron definidos en base a la presencia de las especies en cada levantamiento (Figura 2).

\section{Descripcion de la ecología de la unidad I:}

La unidad I es la mas representada, se caracteriza por una distribución entre 2094 y 2391 msnm.

El segundo nivel del "eigenvalor $=0.435$ " (bajo), separa a la unidad I en tres subunidades; 1.1) estratos briofitas y líquenes, 1.2) estrato herbáceos, 1.3) arbustivo bajo y arbustivo alto. Las especies que registran una regeneración mayor según la escala de Braun-Blanquet, son las especies; Nertera granadensis con $75 \%$ de cobertura, herbácea rastreara, la presencia de esta especie en la unidad I es muy frecuente, seguido por Lycopodium jussiaei con $50 \%$ de cobertura, de igual forma Elleanthus ecuadorensis con $50 \%$ de cobertura se hace presente en diferentes lugares de la caldera, especialmente en sustrato rocoso reciente, al igual Pernettya prostrata con $50 \%$ de cobertura, es un arbusto de 35 a $40 \mathrm{~cm}$ de altura, presente en sitios de regeneración recientes sobre lavas volcánicas y Blechnum divergens con 50\% de cobertura, es un helecho que está disperso en toda las zonas de regeneración reciente (ver la Figura 4).

Esta unidad presenta pendientes entre 5 a $40 \%$, su altitud varía entre 2094 a 2391 msnm, con vista al cono volcánico que se encuentra activo con estruendos frecuentes durante las 24 horas del día, en cuanto al suelo, presenta diferentes porcentajes de suelo desnudo rocoso, suelo de acumulación de lava volcánica o simplemente grandes cantidades de rocas volcánicas sedimentadas.

\section{Descripción de la ecología de la unidad II}

Esta unidad se encuentra entre los 2152 a los 2213 msnm, el primer nivel del "eigenvalor $=0.426$ ", en el lado derecho del dendrograma divide a la unidad II, en única asociacion donde separa de la unidad I en estratos briofitas y líquenes en su mayoría además en estrato herbáceo y arbustivo bajo con poca representatividad en esta unidad. Según la escala de Braun-Blanquet, establece los porcentajes de cobertura a las especies; Pityrogramma calomelanos con 50\% de cobertura, es un helecho pionero que pueden adaptarse en lugares desérticos o de suelo desduno, al igual que la especie Elleanthus fractiflexus, con $50 \%$ de cobertura, es una orquídea que agrupa en lugares desérticos localizadas en las parcelas de 17 al 20, mientras que la especie Gunnera atropurpurea presenta una cobertura con

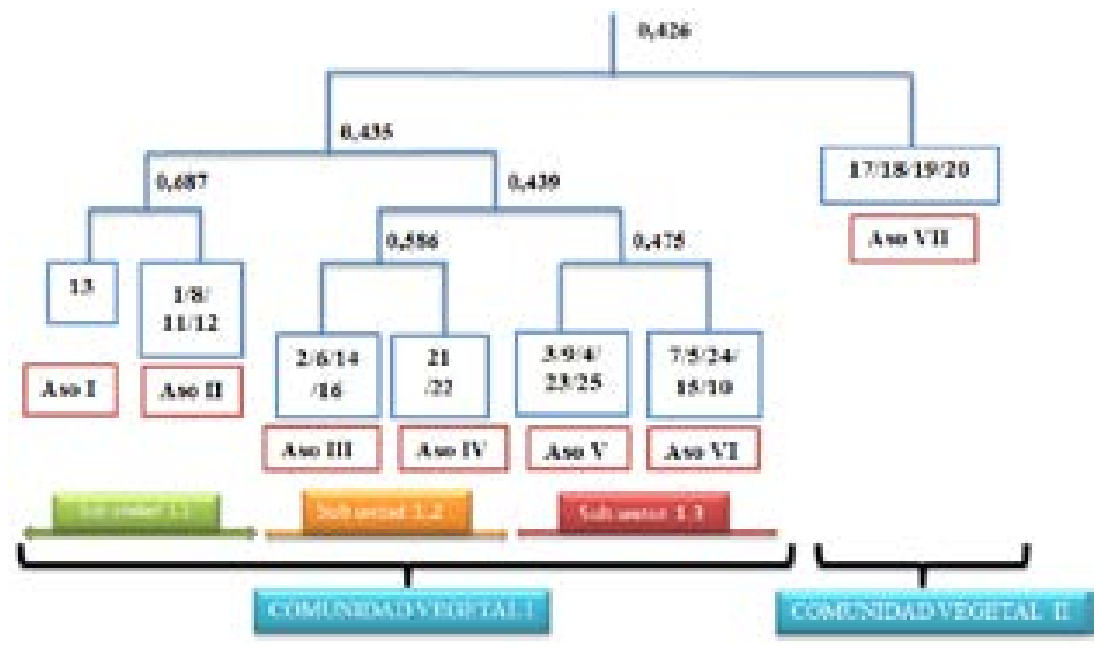

Figura 2. Dendrograma de la agrupación de las 25 parcelas con sus respectivos eigenvalores para cada divisiónsegún análisis Twinspan 
un porcentaje de $25 \%$ en esta unidad como especies pioneras, la familia Ericaceae también se presentan como pionera en lugares desérticos, en especial la especie Disterigma balslevii con 5\% de cobertura, un arbusto de $0.10-0.30 \mathrm{~m}$ de altura, presente en lugares con un buen grado de adaptabilidad. La presencia de esta especie en la unidad II es escasa, mientras que la unidad I es muy frecuente (Ver la Figura 6).

La unidad II corresponde a la zona desértica del lado norte-este del volcán, formado por una erupción reciente (2002), con gran cantidad de rocas volcánicas de diversos tamaños. Esta unidad solo presenta una asociacion que incluye las parcelas 17 al 20 identificada como asociacion VII, el suelo presenta con una pendiente de 20 a $30 \%$ con respeto al nivel más bajo que es $0 \%$ sobre un suelo rocoso de pendiente moderada.

\section{Descripcion de la ecología de la asociacion I y II:}

El clado izquierdo del dendrograma separa a la asociación I y II, en el que se agrupa las parcelas 13, 1, 8,11 y 12 , con un nivel del "eigenvalor $=0.687$ ". Posee vegetación herbácea muy poblada, como indicador de una regeneración primaria positiva, dentro de la caldera, también combinada con especies arbustivos, en este sitio de estudio presenta un rango de cobertura vegetal del 95 al 100\%. Según la escala de Braun-Blanquet, establece los valores de abundancia a las especies; Nertera granadensis con $75 \%$ de cobertura, seguidos con una dominante cobertura por arbustos de especies: Macleania rupestris (50\%), Parodiolyra lateralis (25\%), Pityrogramma calomelanos (25\%), Blechnum divergens (5\%) y Gunnera brephogea (5\%), (Ver la Figura 8). Datos de diversidad se muestran en la Cuadro 5. El rango altitudinal de distribución de la asociación I y II se da sobre los 2094 - 2132 msnm, en una pendiente del 5 al $15 \%$, esto determina que el lugar es muy favorable para regeneración de diferentes especies por sus características del suelo que está formado por arena y cenizas volcánicas; además en este tipo de suelos con poca pendientes tiene a su favor en retención de agua y muchas nutrientes que son arrastradas desde la parte alta del cono volcánico.

Descripción de la ecología de la asociacion III y IV.

Esta asociación se encuentra entre 2113 y 2391 msnm, las parcelas $2,6,14,16,21$ y 22 estan incluiodads en asociación vegetal.

Hacia el clado izquierdo se separa las asociaciones vegetales III, IV, V y VI con un nivel del "eigenvalor $=0.439$ ", de lo cual desprende la asociacion III y IV con un nivel del "eigenvalor $=0.586$ ". Lo que indica que poseen una afinidad media - alta en su composición florística. Las especies pioneras que registran una sucesión mayor según la escala de Braun-Blanquet son: Macleania rupestris con cobertura de 50\%, Monocheatum lineatum con 50\% Gunnera brephogea con $50 \%$ de cobertura, Erato polymnioides con $25 \%$, Elleanthus ecuadorensis con $25 \%$, esta última especie tiene un grado de adaptabilidad muy favorable en ambientes con poca presencia de materia orgánica (Ver la figura 9), en el cuadro 6 se muestran datos de diversidad. Se desarrolla en un pendiente que van de $1 \%$ a $30 \%$, lo cual demuestra un suelo regular ya que varía de su distribución de especies en diferente zonas de topografía agreste, el lugar favorece en sucesión de especies ya que en años demuestra que no ha sido perturbado el lugar, eso da una señal de recuperación avanzada de las asociaciones vegetales.

\section{Descripcion de la ecología de la asociacion V y VI:}

Esta asociación se encuentra entre 2090 hasta los 2208 msnm. Hacia el clado izquierdo separa a la asociación vegetal III, IV, V y VI con un nivel del "eigenvalor = 0.439", de lo cual desprende la asociacion V y VI con un nivel del "eigenvalor $=0.475$ ". Esta unidad de paisaje agrupa las parcelas 3, 4, 9, 23, 25 у 5, 7, 10, 15, 24, con una cobertura continua de la vegetación del $100 \%$. Según la escala de Braun-Blanquet, las especies que mas dominan en estas parcelas son: Lycopodium jussiaei con $75 \%$ de cobertura, Elleanthus ecuadorensis con $50 \%$, Blechnum divergens con $50 \%$, Erato vulcanica con $25 \%$, Pernettya prostrata con $25 \%$, Pitcairnia alata con $25 \%$, Gunnera atropurpurea con $25 \%$, estas especies se adaptan como especies pioneras con más éxito de supervivencia en suelos poco desarrollado (Ver figura 12). Presenta una pendiente de $1 \%$ al $40 \%$ de relieve, lo que determina que la topografía dentro de la caldera del volcán es moderada, con características abióticas similares a las otras unidades. Datos de diversidad ver Cuadro 7.

\section{Endemismo en la caldera del volcán Reventador}

Las 12 especies endémicas encontradas corresponden a un endemismo de tipo nacional; las principales familias por número de especies son: Bromeliaceae (3) y Melastomataceae (2), las otras familias con una sola especie, en total nueve familias en especies suman doce, siendo un alto grado de endemismo en base a la superficie muestreada.

En la caldera del volcán Reventador el estado de conservación según los criterios establecidos por la UICN son lo siguiente: cuatro endémicas son catalogados En Peligro, tres endémicas Vulnerables, dos endémicas en Preocupación Menor, un endémica Casi Amenazada, un endémica No Evaluada, un endémica Datos Insuficientes, además seis de catorce endémicas no incluyen hallazgos dentro del SNAP 


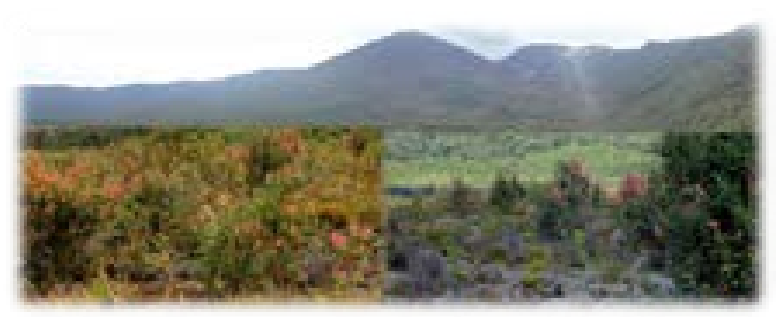

Figura 3. Vista general de la unidad I, la imagen de izquierda representa población de la familia Ericaceae, imagen derecha poblada de arbustos y rocas con liquines

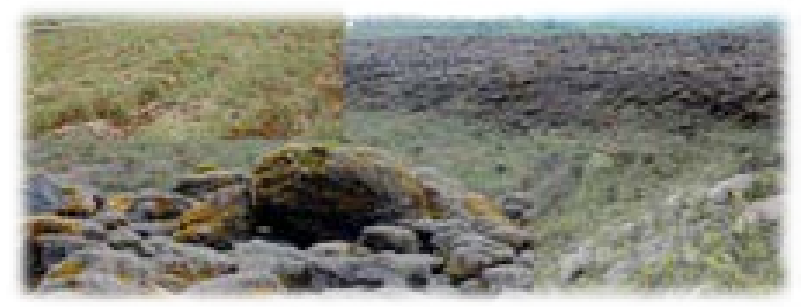

Figura 5. Vista general de la unidad II, formado por paisajes de erupción reciente con gran cantidad de rocas y sobre ella predomina líquenes, musgos, elechos y algunas Orquídeas y Ericaceae

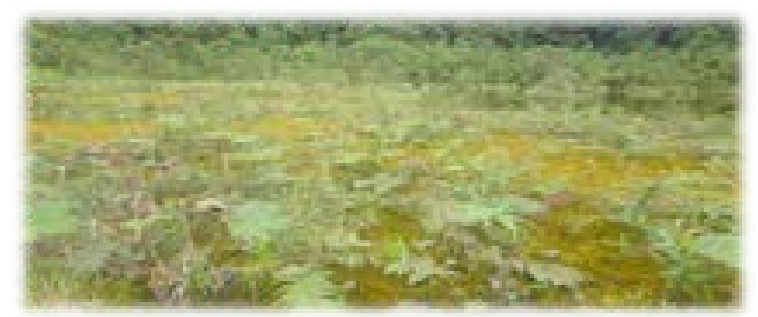

Figura 7. Vista general de la asociación vegetal I y II, presencia de musgos y herbaceaes representativas de Gunnera, helechos y algunas Asteraceae

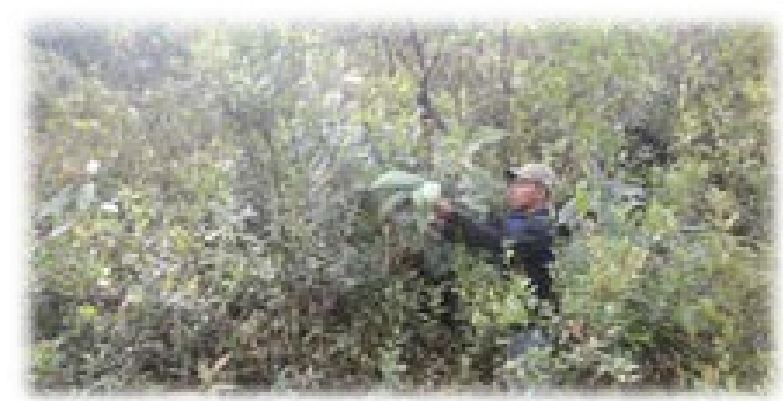

Figura 9. Vista general de la asociación vegetal III y IV, formada por arbustivos altos es una zona de erupción antigua, con regeneración natural avanzada

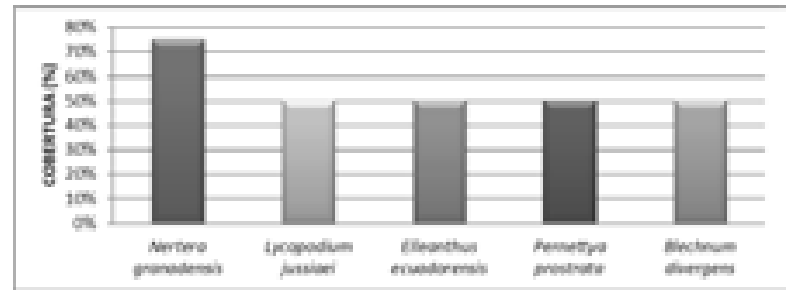

Figura 4. Cinco especies con mayor regeneración según la escala de Braun-Blanquet (1979).

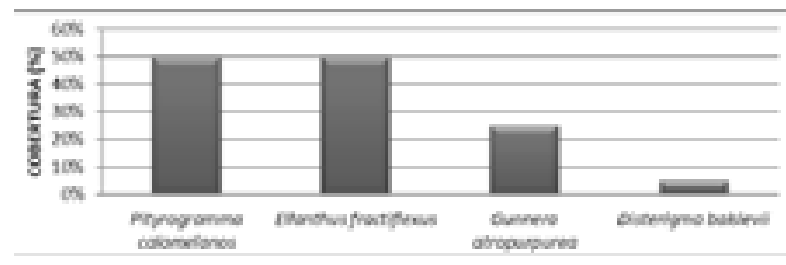

Figura 6. Especies con mayores coberturas según la escala de Braun-Blanquet (1979)

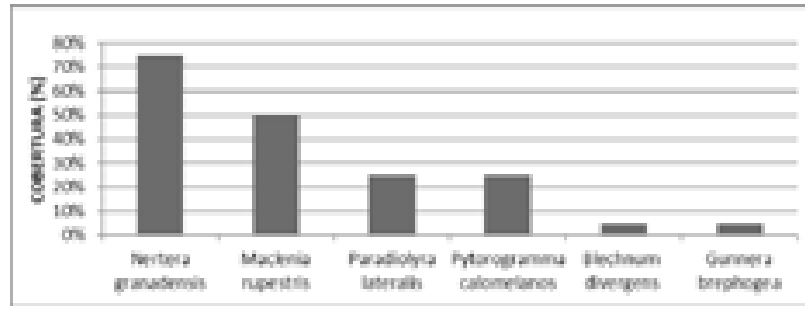

Figura 8. Especies con mayores cobertura según la escala de Braun-Blanquet (1979). presentes en la asociación vegetal I y II

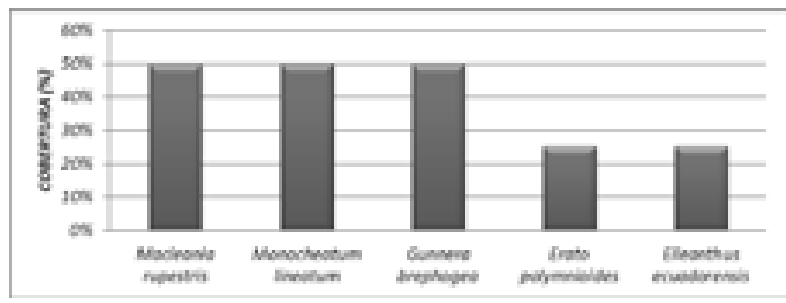

Figura 10. Especies con mayores coberturas según Braun-Blanquet, presentes en la asosacion vegetal III y IV 


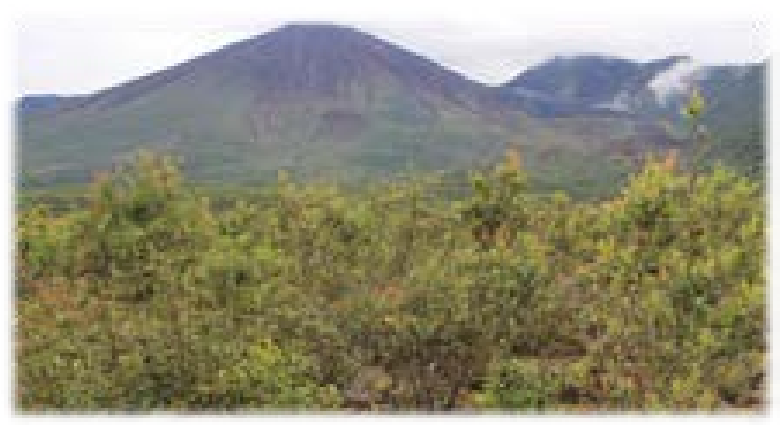

Figura 11. Vista general de la asociación vegetal V y VI, formado por estrato arbustivo bajo en ella predomina la familia Ericaceae y sobrecelan algunas orquídeas

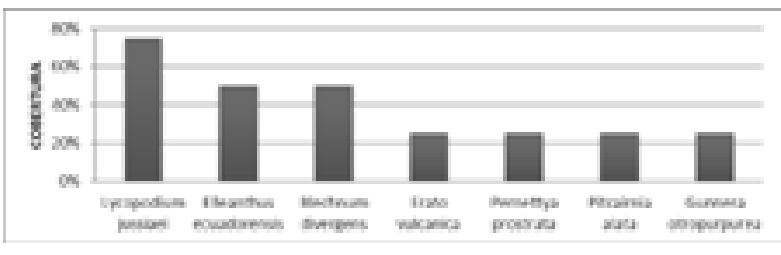

Figura 12. Especies con mayores coberturas según la escala de Braun-Blanquet (1979), presentes en la asosacion vegetal $\mathrm{V}$ y $\mathrm{VI}$

Cuadro 3. Diversidad relativa $(\mathrm{DiR})$ y riqueza florística de la especie más dominante de la unidad $\mathrm{I}$.

\begin{tabular}{lccl}
\hline \multicolumn{1}{c}{ Familia } & $\begin{array}{c}\text { No } \\
\text { Especies }\end{array}$ & DiR (\%) & \multicolumn{1}{c}{ Más representativas } \\
& & & \\
\hline Orchidaceae & 19 & 16.96 & Elleanthus ecuadorensis \\
Asteraceae & 8 & 7.14 & Liabum kingii \\
Poaceae & 8 & 7.14 & Parodiolyra lateralis \\
Bromeliaceae & 7 & 6.25 & Pitcairnia alata \\
Ericaceae & 7 & 6.25 & Pernettya prostrata \\
Melastomataceae & 6 & 5.36 & Monocheatum lineatum \\
Rubiaceae & 6 & 5.36 & Nertera granadensis \\
Blechnaceae & 4 & 3.57 & Blechnum divergens \\
Dryopteridaceae & 3 & 2.68 & Elaphoglossum ambiguum \\
Lycopodiaceae & 3 & 2.68 & Lycopodium jussiaei \\
Gunneraceae & 2 & 1.80 & Gunnera brephogea \\
\hline
\end{tabular}

Cuadro 4. Diversidad relativa (DiR) y riqueza florística de la unidad vegetal II

\begin{tabular}{lccl}
\hline \multicolumn{1}{c}{ Familia } & $\begin{array}{c}\mathbf{N}^{\circ} \\
\text { Especies }\end{array}$ & DiR (\%) & Mas representativas \\
\hline Ericaceae & 3 & 37.50 & Disterigma balslevii \\
Orchidaceae & 2 & 25.00 & Elleanthus fractiflexus \\
Gunneraceae & 1 & 12.50 & Gunnera atropurpurea \\
Pteridaceae & 1 & 12.50 & Pityrogramma calomelanos \\
\hline
\end{tabular}


Cuadro 5. Diversidad relativa (DiR) y riqueza florística de la asociación I y II

\begin{tabular}{lccl}
\hline \multicolumn{1}{c}{ Familia } & $\mathbf{N}^{\circ}$ Especies & DiR (\%) & \multicolumn{1}{c}{ Más representativas } \\
\hline Poaceae & 6 & 13.95 & Parodiolyra lateralis \\
Ericaceae & 5 & 11.63 & Macleania rupestris \\
Rubiaceae & 5 & 11.63 & Nertera granadensis \\
Gunneraceae & 2 & 4.65 & Gunnera brephogea \\
Blechnaceae & 1 & 2.32 & Blechnum divergens \\
Pteridaceae & 1 & 2.32 & Pityrogramma calomelanos \\
\hline
\end{tabular}

Cuadro 6. Diversidad relativa (DiR) y riqueza florística de la asociación vegetal III y IV

\begin{tabular}{lccl}
\hline \multicolumn{1}{c}{ Familia } & $\begin{array}{c}\text { No } \\
\text { Especies }\end{array}$ & DiR (\%) & \multicolumn{1}{c}{ Mas representativas } \\
\hline Ericaceae & 6 & 10.91 & Macleania rupestris \\
Asteraceae & 5 & 9.09 & Erato polymnioides \\
Orchidaceae & 5 & 9.09 & Elleanthus ecuadorensis \\
Melastomataceae & 4 & 7.27 & Monocheatum lineatum \\
Rubiaceae & 3 & 5.45 & Galium corymbosum \\
Cyperaceae & 2 & 3.63 & Rhynchospora polyphylla \\
Gunneraceae & 2 & 3.63 & Gunnera brephogea \\
\hline
\end{tabular}

Cuadro 7. Diversidad relativa ( DiR) y riqueza florística más dominante de la asociación $\mathrm{V}$ y VI

\begin{tabular}{lccl}
\hline \multicolumn{1}{c}{ Familia } & $\begin{array}{c}\mathbf{N}^{\circ} \\
\text { Especies }\end{array}$ & DiR (\%) & \multicolumn{1}{c}{ Más representativas } \\
\hline Orchidaceae & 16 & 19.05 & Elleanthus ecuadorensis \\
Asteraceae & 8 & 9.52 & Erato vulcanica \\
Ericaceae & 7 & 8.33 & Pernettya prostrata \\
Bromeliaceae & 6 & 7.14 & Pitcairnia alata \\
Lycopodiaceae & 3 & 3.57 & Lycopodium jussiaei \\
Blechnaceae & 2 & 2.38 & Blechnum divergens \\
Gunneraceae & 2 & 2.38 & Gunnera atropurpurea \\
\hline
\end{tabular}




\section{Cuadro 8. Especies endémicas del Ecuador registradas en el interior de la caldera del volcán Reventador}

\begin{tabular}{ll}
\hline Especies endémicas & Características \\
\hline
\end{tabular}

Oreopanax avicenniifolius (Kunth) Decne. \& Planch.

Liabum kingii H. Rob.

Guzmania fusispica Mez \& Sodiro

Pitcairnia alata L.B. Sm.

Werauhia paupera (Mez \& Sodiro) J.R. Grant

Macleania subsessilis

Luteyn

Axinaea pauciflora Cogn.

Axinaea sessilifolia Triana

Elleanthus ecuadorensis

Garay

Pennisetum pauperum Nees ex Steud.

Roupala brachybotrys

I.M. Johnst.

Prunus herthae Diels
Arbusto o árbol endémica de los andes 2500 - 3500 msnm. Estado de connservacion $=$ Casi amenazada

Sub-arbusto endémica de los andes se registra sobre 1000 - 2000 - 3000 msnm. Estado de connservacion = Preocupacion menor.

Hierba epifita endémica de los andes va de 1000 - $2000 \mathrm{msnm}$. Estado de connservacion $=$ vulnerable

Epifita de los andes sobre los 2000 - 3000 msnm. Estado de connservacion $=$ En peligro.

Epifita registrado de $0-1000 \mathrm{msnm}$. Estado de connservacion de especies según UICN: VU A4c = Vulnerable.

Arbusto de la costa y andes $0-1500 \mathrm{msnm}$. Estado de connservacion $=$ En peligro.

Arbusto o árbol de los andes 2000 - $2500 \mathrm{msnm}$.

Estado de connservacion $=$ En peligro

Árbol de los andes va de 1500 - 3000 msnm.

Estado de connservacion $=$ Vulnerable .

Herbácea de la costa y andes van de 500 - 2500 msnm. Estado de connservacion $=$ Preocupación menor.

Herbaceae de galápagos y andes va desde 1000 - 2000 msnm. Estado de connservacion $=$ No evaluada.

Arbusto o árbol de los andes 1000 - $2500 \mathrm{msnm}$. Estado de connservacion $=$ No peligro .

Árbol de los andes y amazonia va desde 0 - 500 y $1000-1500$ msnm. Estado de connservacion $=$ datos insuficientes, 


\section{Conclusiones}

$\mathrm{S}$ eencontró dos unidades vegetales y siete asociaciones florísticas, que se destacan como características, agrupadas en diferentes estratos vegetales: herbáceo, arbustivo alto y bajo, suelos desnudos poblados por líquenes y musgos. El área corresponde aun área antes no estudiada. Se registraron 112 especies de plantas vasculares, pertenecientes a 70 géneros en 42 familias botánicas, de estos, 43 géneros $(61.43 \%)$ contienen una sola especie, 20 géneros (28.57\%) están representados por dos especies, y 7 géneros $(10 \%)$ por mas de dos especies. Las familias más diversas de la flora en el interior de la caldera que se registraron son Orchidaceae con 19 especies (16.96\%) que abarca gran cantidad de niveles de sucesión ecologica, Asteraceae y Poaceae con 8 especies $(7.14 \%)$ respectivamente, Ericaceae y Bromeliaceae con 7 especies $(6.25 \%)$ predominante, Blechnaceae con 4 especies (3.57\%), Clusiaceae, Cyperaceae, Dryopteridaceae y Lycopodiaceae con 3 especies $(2.68 \%)$ respectivamente, mientras que los géneros con mayor diversidad relativa son: Epidendrum con 8 especies (7.27\%), Elleanthus con 5 especies (4.46\%), Blechnum con 4 especies $(3,57)$, Elaphoglossum, Lycopodium, Axinea y Psychotria con 3 especies $(2.69 \%)$ respectivamente.

Segun estudios realizados por Schaefer (2008) en sucesión primaria de la vegetación sobre los flujos piroclásticos (FPs) del 2006 en el volcán Tungurahua, lo complejo de estudios con impacto volcánico y humano, que atribuyen a materiales piroclasticos y sugieren que la mayoría de las plantas no aparecen porque el recrecimiento de los bancos de semilla en el suelo no son viables, afirmando que las plantas llegan al flujo, por medio abiótico como por ejemplo el viento o bióticos tales como animales, esta explicación tendría concordancia ya que la influencia del bosques presente alrededor de la caldera están jugando un papel muy importante en regeneración natural en el interior de la caldera.

Con el presente modelo se intenta explicar los procesos que se dan en los cambios sucesionales, los factores que los regulan y los mecanismos que operan a nivel de especies vegetales. Se explica el concepto de etapa y el proceso de cambio entre una etapa y la siguiente, las diferentes condiciones microclimáticas, de suelos y las perturbaciones causadas por la actividad volcánica, son los factores que junto a las características adaptativas de cada especie, generan un mosaico de diferentes asociaciones vegetales.

De acuerdo a la agrupación de elementos como condiciones climáticas y geográficas, régimen de perturbaciones, grado de adaptación de los organismos colonizadores a las condiciones del sitio, y competencia e interacción entre organismos, se puede representar la forma en que funciona la regeneración en la caldera:

1. Luego de la perturbación por la actividad volcánica, se crea un nuevo terreno sin vegetación (sustrato estéril en este caso), el cual está disponible para las cianobacterias y para una recolonización de especies vegetales (musgos y briofitas).

2. Algunas especies de áreas cercanas al área perturbada inician la colonizacion de diferentes formas, dispersión de semillas por el viento o por aves, o propágulos vegetativos si han sobrevivido en el suelo.

3. Las plantas invasoras mueren o se establecen depende de su condición adaptativa en un nuevo sustrato.

4. Las interacciones de competencia y mutualismo entre las plantas afectan su establecimiento y posterior desarrollo.

5. Simultáneamente, las plantas producen variaciones en las condiciones del sitio por efecto de; luz, temperatura y propiedades del suelo, hecho que puede, o no, afectarlas en su continua interacción con otras plantas y animales.

En el caso de la caldera del volcán Reventador, en base al análisis de la vegetación muestreada, se pudieron identificar cuatro fases o etapas de la sucesión primaria, la cual inicia con la sobreposición de lava (sustrato estéril) en la vegetación existente, proceso denominado por (Walker y Del Moral, 2003) como nudación o denudación. Luego de la migración y establecimiento de organismos que toleran las condiciones del sitio, tales como algas y líquenes, se inicia el proceso en el cual se crean las condiciones necesarias para el establecimiento de especies pioneras que se dispersan hacia el sitio. La colonización de especies se inicia a partir de la dispersión de semillas por el viento o por la fauna existente en el sitio. Si ocurren actividades fumarólicas, es posible que estas provoquen una regresión a la etapa inicial de la regeneración.

Debido a que el modelo propuesto en el presente estudio se basa en la comparación de la vegetación de diferentes sitios que se supone, son de diferentes edades, ya que la última erupción de gran magnitud se presentó en el año 2002 a partir de esa fecha ha surgido pequeñas erupciones alcanzando perturbaciones en la caldera a diferentes magnitudes, por lo tanto se asume que las condiciones iniciales de sitio en los estratos de herbáceo, arbustivo bajo y alto son similares, también presenta suelo desnudo formado por lavas y rocas volcánicas, donde prevalecen líquenes y musgos.

Al comparar los estudios de Schaefer (2008), se obserava que la regeneración natural sobre los campos de 
material piroclásticos del volcán Tungurahua presentan menor representatividad de elementos florísticos que la caldera del volcán Reventador, 47 especies frente a 112 especies, con una notable presencia de doce especies endémicas, mientras que datos del volcán Sangay en altitudes entre los 3500 a 4390 msnm señalan un endemismo de siete especies en un total de 126 especies registradas en la gradiente altitudinal (Lozano et. al. 2015).

Aguirre (2001) explica que la diversidad y composición florística de una área de vegetación disturbada por incendios forestales, donde se han registrado 137 especies dentro de 109 géneros en 50 familias, entre los 2250 a $2520 \mathrm{msnm}$, la comparación con el registro de la caldera del volcán Reventador en análisis de estos datos nos da un escenario casi similar en ambos estudios con la diferencia que el uno es producto de un incendio forestal y la otra producto de erupciones volcánicas, el endemismo encontrado por Aguirre (2001), muestra 15 especies endémicas, indicativo de un alto endemismo para ambos escenarios.

Se establece que los ecosistemas de la caldera del volcán Reventador y su alrededor, no se evidencio ninguna actividad humana, tales como la quema, agricultura, pastoreo, tala de árboles, etc. La caldera del volcán Reventador es un caso aislado de toda actividad humana, se encuentran en un estado medio de conservación ya que su permanencia está amenazado por la constante actividad del volcán Reventador, cabe señalar, que es uno de los volcanes más activos del Ecuador, dentro de la caldera presenta un rango de cobertura vegetal de 70 a $100 \%$. La conservación de este ecosistema es debido a la distancia, terreno agreste y al difícil acceso al lugar y principalmente por una constante amenaza del volcán Reventador convirtiéndose en un área optima de investigación de los procesos de sucesión vegetal y cambios de la estructura en el tiempo.

\section{Agradecimientos}

Tos autores del presente trabajo agradecen al - - Ministerio del Ambiente-Chaco a su director y técnicos, así como a la Universidad Estatal Amazónica.

\section{Bibliografía}

Aguirre, Z. 2001. Diversidad y composición florística de un area de vegetación disturbada por incendios forestales. Educación Ambiental y Recreación Ing. Francisco Vivar C. PUEAR, de la Universidad Nacional de Loja-Ecuador.

Beard, L.S. 1945. The progress of plant succession on the Soufriere of St. Vincent. Ecology 32: 1-9.

Braun Blanquet, J. 1979. Fito Sociología-Bases para el Estudio de las Comunidades Vegetales. España.
Eggler, W.A. 1959. Manner of invasion of volcanic deposits by plants, with further evidence from Paracutin and Jorullo. Ecol. Monogr. 29: 267-286.

Hall, M., Ramón, P., Monthes, P., LePennec, J.L., Garcia, A., Samaniego, P., Yepes, H. 2004. Volcanic eruptions with little warning: the case of Volcán Reventador's Surprise November 3, 2002 Eruption, Ecuador. Revista Geológica de Chile, 31(2): 349-358.

Hill, M.O. \& P. Smilauer. 2005. TWINSPAN for windows version 2.3. Center for Ecology and Hydrology \& University of South Bohemia, Huntingdon \& Ceske Budejovice. $29 \mathrm{p}$.

Lozano, P., Duque, J., Balslev, H., Ollgaard, B, Peyre, G, Vallejo, S., Valverde, V. \& P. López. 2015. Flora Paramuna del la Base del Cono del Volcan Sangay Amazonía del Ecuador. Universidad Estatal Amazónica. Puyo Ecuador. Pp. 135.

Ministerio del Ambiente del Ecuador, 2013. Sistema de Clasificación del Ecuador Continental. Subsecretaria de Patrimonio Natural, Quito.

Penzing, O. 1902. Die Fortschritte des Flora des Krakatau. AM. Jard. Bot. Buitenzorg, 8: 92-115.

Schaefer, L. 2008. La sucesion primaria de la vegetacion sobre los flujos piroclásticos del 2006 en el Volcan Tungurahua. Trabajo no publicado, De Pauw University, EEUU. 25p

Skotberg, C. 1941. Plant succession and recent lava flows in the istand of Hawaii. Goteborgs Kungl. Vetens Kapsoch. Vitterherts-Samhiilles Handdlingar, Sjatte roljden. Ser B, Bandkt, 8: 1-32.

Valencia, R., Pitman, N., Leon-Yanes, S. y Jørgensen, P. 2000. Libro Rojo de las Plantas Endémicas del Ecuador. Herbario QCA. Pontificia Universidad Católica del Ecuador, Quito.

Vargas, U., G. 1985. La sucesión vegetal primaria en una región de vulcanismo reciente en el Volcán Arenal y sus alrededores, Costa Rica. Departamento de Geografía, Universidad de Costa Rica. 13 p.

Walker, L.R., R. del Moral. 2003. Primary succession and ecosystem rehabilitation. Cambridge University Press, UK. 456 p.

Ciencia y Tecnología. 2020. 13(1):27-37 